\title{
Predictors of Mechanical Failure Rate in Reduction of Intertrochanteric Fracture of the Femur Using Proximal Femoral Nail with Helical Blade
}

\begin{abstract}
Background: The optimal implant for reducing intertrochanteric fracture is unknown. Several bone-related, implant-related and radiological variables seem to play a role in predicting the rate of intraoperative and postoperative mechanical failure. The objective of this study was to evaluate the rate and predictors of mechanical failure and its relationship with quality of reduction through assessing certain radiological parameters.
\end{abstract}

Materials and methods: A retrospective study reviewed patients with stable (AO/OTA Type 3.1A1) and unstable (AO/OTA Type 3.1A2 and $A 3$ ) intertrochanteric femur fracture who were treated with proximal femoral nail with helical blades (PFNA) between 2011 and 2017. Exclusion criteria were patients with pathologic fractures. Followup was undertaken at 1 day, $4-6$ weeks, and 3 months postoperatively by reviewing radiographs and medical charts. The primary outcome was the rate of mechanical failure. Radiologic parameters of Tip apex distance (TAD), neck shaft angle and displacements were evaluated as indicators of reduction quality. All these were compared to each other and baseline patient characteristics to identify any association.

Results: We found 69 patients who underwent PFNA procedure. There was no case of intraoperative or postoperative mechanica failure or non-orthopedic complication. The postoperative radiological outcomes revealed variable quality of reduction with: mean TAD of $26.07 \pm 3.69^{\circ}$ (bimodal), mean neck shaft angle of $133 \pm 5.6^{\circ}$ (range: $116-140^{\circ}$ ), $35 \%$ of patients having lesser trochanter displacement, $28 \%$ having displaced femoral shaft. There was no statistical significant relation between any of the radiological outcomes and patien characteristics except between neck shaft angle and osteoporosis $(\mathrm{p}=0.03)$.

Conclusion: The radiological outcomes are independent of the patient's characteristics except for Neck Shaft Angle and Osteoporosis. Absence of mechanical failure despite the variability in reduction quality (optimal/suboptimal) might be explained by the decent implant design. Further studies are needed to confirm that PFNA implant design is the major preventer of mechanical failure.

Level of evidence: IV

Keywords: Intertrochanteric fracture; Femur; Mechanical failure: Proximal femoral nail with helical blade; Radiological outcomes

\section{Introduction}

During the past few decades, the incidence of intertrochanteric femoral fracture raised due to the significantly increased life expectancy [1]. It is estimated that the incidence of intertrochanteric fracture will double in 2040 [2]. Elderly patients with this fracture are recommended to be treated with a minimally invasive operation, which can ensure optimal reduction and assembly with fewest intraoperative and postoperative complications and lowest mechanical failure rate.

Different cephalomedullary implant designs were used with different radiological, functional and operative outcomes. Many studies tried to compare the intraoperative and postoperative

\section{Journal of}

Orthopedics \& Rheumatology

\author{
Ahmad Salaheddine Naja ${ }^{1 *}$, Tammam Hanna ${ }^{1}$, \\ Rachid Haidar ${ }^{1}$, Hani Tamim² and Said Saghieh ${ }^{1}$ \\ ${ }^{\prime}$ Orthopedic Surgery Division, American University of Beirut Medi- \\ cal Center, Lebanon \\ ${ }_{2}^{2}$ Internal Medicine Department, American University of Beirut \\ Medical Center, Lebanon \\ *Address for Correspondence \\ Ahmad Salaheddine Naja, Orthopedic Surgery Division, American \\ University of Beirut Medical Center, Beirut, Lebanon, Tel: +961 3201173; \\ Email: ahmadnaja90@hotmail.com \\ Submission: 2 April, 2018 \\ Accepted: 04 May, 2018 \\ Published: 14 May, 2018 \\ Copyright: (๑) 2018 Naja AS, et al. This is an open access article \\ distributed under the Creative Commons Attribution License, which \\ permits unrestricted use, distribution, and reproduction in any medium, \\ provided the original work is properly cited.
}

orthopedic complications as well as non-orthopedic complications of intertrochanteric fracture reduction using different types of cephalomedullary implants. Nevertheless, due to lack of sufficient randomized clinical trial and the multiplicity variables in predicting the rate of mechanical failure, results about the optimal implant for fixing intertrochanteric fracture are still conflicting.

Mostly, two options are used: Dynamic hip screw (DHS) and intramedullary implants [3]. Proximal femoral nail with helical blade intramedullary nail (PFNA Synthes ${ }^{\mathrm{rn}}$ Switzerland) is frequently used for fixation of intertrochanteric fracture. It has a helical blade rather than a leg screw for better attachment in the femoral head. Reduction of intertrochanteric fracture can be optimal or suboptimal with PFNA technique as evident in postoperative radiographic parameters like tip apex distance (TAD), neck shaft angle, displacement of the femoral shaft and the lesser trochanter.

Many studies have compared different radiological parameters for assessing the rate of mechanical failure [4-7]. Tip Apex Distance (TAD) is considered one of the strongest predictors of cut-out using intramedullary nails [8]. Displacement of the femoral shaft is another important indicator of cut-out rate [9]. However, to the best of our knowledge, no study has yet analyzed the relationship between patients' characteristics, radiological parameters and the rate of implant failure using proximal femoral nail with helical blade (PFNA). Here, we aim to identify the rate of mechanical failure in these patients and also measure the post-operative radiographic parameters that evaluate quality of reduction in order to find any relation between quality of reduction and mechanical failure.

\section{Materials and Methods}

This was a retrospective observational study done in a single tertiary care center. The approval of the institutional review board (IRB) was secured through our institution office which follows Declaration of Helsinki/HIPPA regulations. All the patients aged 50 
Citation: Naja AS, Hanna T, Haidar R, Tamim H, Saghieh S. Predictors of Mechanical Failure Rate in Reduction of Intertrochanteric Fracture of the Femur Using Proximal Femoral Nail with Helical Blade. J Orthopedics Rheumatol. 2018; 5(1): 5

and above who had undergone intertrochanteric fracture reduction using a PFNA between 2011 and 2017 were included. We excluded patients who had neurologic disease, poliomyelitis, cerebro vascular accident affecting ipsilateral or contralateral lower extremity and those with pathological and/or open fractures.

The following data was gathered from the medical records: age, gender, co-morbidities, presence of osteoporosis, American Society of Anesthesiologist (ASA) score, and fracture-related information which includes the laterality and type of fracture (stable/unstable) of the intertrochanteric fracture. Unstable fracture was defined according to the AO-Muller/Orthopedic Trauma Association classification as fracture 31-A2 and 31-A3.

The outcome measures assessed were: the quality of reduction and the postoperative rate of mechanical failure. To evaluate the reduction level, the authors re-read the post-operative radiographs of these patients and measured the TAD, neck shaft angle, displacement of the femoral shaft and displacement of the lesser trochanter. The presence of TAD $>25 \mathrm{~mm}$, neck shaft angle between $125-135^{\circ}$ and nondisplaced femoral shaft or lesser trochanter is considered an optimal reduction. The radiographs and medical charts were reviewed for postoperative period to identify callus formation or fracture healing in addition to any intraoperative complication and postoperative mechanical failure like cut-out and non-union.

\section{Surgical procedure}

Proximal femoral anti-rotation: PFNA system (Synthes ${ }^{\mathrm{Tx}}$, Switzerland) was approved by AO/ASIF in 2004 (Figure 1). The distinctive feature of the implant is the use of a single blade with a large surface area (Figure 1). The nail used in our study is $240 \mathrm{~mm}$ in length, $10 \mathrm{~mm}$ in diameter, $130^{\circ}$ neck shaft angle with a $6^{\circ}$ mediolateral angle proximally and distally. Distal locking was static in all patients.

Operative technique: Surgery was carried under spinal or general anesthesia. The patient was laid in supine position with the unaffected leg abducted and placed on a leg support. Reduction was done by traction and manipulation. A $5 \mathrm{~cm}$ incision was done proximal to the greater trochanter and deepened through the subcutaneous tissue until reaching the fascia which was incised along the wound. The PFNA system Owl synthesis was inserted at the middle aspect of the greater trochanter and advanced through the proximal canal A long guide wire was inserted and made sure to be intra-femoral down to the knee. Proximal reaming started with $8.5 \mathrm{~mm}$ diameter reaming head, and we increased to a diameter of 0.5 to 1.5 greater than the nail diameter. Then, approximal femoral nail form synthes size $240 \mathrm{~mm}$ was inserted and a new guide wire was inserted through the golden drill sleeve into the bone and confirmed by AP and lateral fluoroscopy. An aiming jig was secured after the insertion of an antirotation wire in the femoral head. The position of the guide wire was verified in anteroposterior (AP) and lateral view. The exact length of the blade was measured. PFNA blade was inserted by applying gentle blows with a hammer. One distal locking screw was inserted into the static hole under fluoroscopy. The final position of the blade nail was checked by AP, and if need be also lateral, radiographs.

\section{Statistical analysis}

The collected data were cleaned and analyzed using SPSS software (version 24, SPSS Statistical Package Inc. USA). Descriptive analyses were performed for all outcomes as mean \pm SD (standard deviation) or frequencies and percentages, as appropriate. Data were analyzed using Chi-Square for categorical variables and student t-test or ANOVA for continuous variables. Also, the cluster classification of some radiographic parameters was assessed and the correlation was calculated using the Pearson coefficient between radiographic parameters and the patient characteristics. A p-value of $<0.05$ indicated statistical significance for all tests.

\section{Results}

One hundred twenty patients had intertronchanteric femoral fracture; however of these, only 69 patients were found to have undergone reduction for intertrochanteric fracture with PFNA and met the inclusion criteria. Six patients were excluded due to pathologic fracture, 25 patients did not have a follow-up lateral $\mathrm{x}$-ray in the hospital data base and 20 patients were lost to follow-up due to death or financial reasons (Figure 2). The average age of the patients was $80 \pm 10$ years (range: $55-90$ years of age) of whom $60 \%$ were females. The average pre-operative weight of these patients was $72.1 \pm 15.6 \mathrm{~kg}$ (range: $40-117 \mathrm{~kg}$ ) and $36.7 \%$ of them were smokers. More than half of the patients had a comorbidity. Diabetes Mellitus type $2(\mathrm{DM})$ was found in 30 patients (44\%), Dyslipidemia (DL) in 24 patients (35\%), and Chronic Artery Disease (CAD) in 22 patients (32\%). Hypertension (HTN) was the most common disease, noted in 39 patients (57\%) and renal disease was the least common, with only 5 patients (7\%). Of the 69 patients, thirteen (19\%) had been previously diagnosed with osteoporosis. The most common ASA was 3 (61\%) followed by $4(22 \%)$ and then $2(10 \%)$.

As for the laterality of the studied intertronchanteric fractures, there were 32 right fractures (46\%) and 37 left fractures (54\%). The most common type of fracture was the unstable one with a frequency of 41 (59\%). All the fractures enrolled were due to a low-energy injury.

The postoperative radiological outcome used to assess the quality of reduction were: Tip apex distance (TAD) classified into two cluster at $23.44 \pm 1.19 \mathrm{~mm}$ and $29.89 \pm 2.35 \mathrm{~mm}$, respectively, neck shaft angle with a mean of $133 \pm 5.6^{\circ}$ (range: $116^{\circ}-140^{\circ}$ ), lesser trochanter displacement was noted in 24 patients (35\%), and displacement of the femoral shaft was noted in 19 of the patients (28\%). When comparing the radiological outcomes with the baseline patient characteristics, there was no statistical significance between any of these radiological outcomes and the patient characteristics except for a relation between neck shaft angle and osteoporosis ( $p$-value $=0.03$ ).

There were no intraoperative complications like femoral shaft fracture. In addition, there was no evidence of any case of nonorthopedic complications like infection, deep vein thrombosis, wound dehiscence, pulmonary embolism, and urinary tract infection for follow-up period of 2.5 months (ranging from 1 month to 6 months). Markers of mechanical failure such as cut-out and nonunion were not noted in any of the patients either.

\section{Discussion}

The introduction of the intramedullary nail system changed significantly the surgical approach for intertrochanteric fracture. It was used as a replacement of the sliding hip screw and aside plate that 


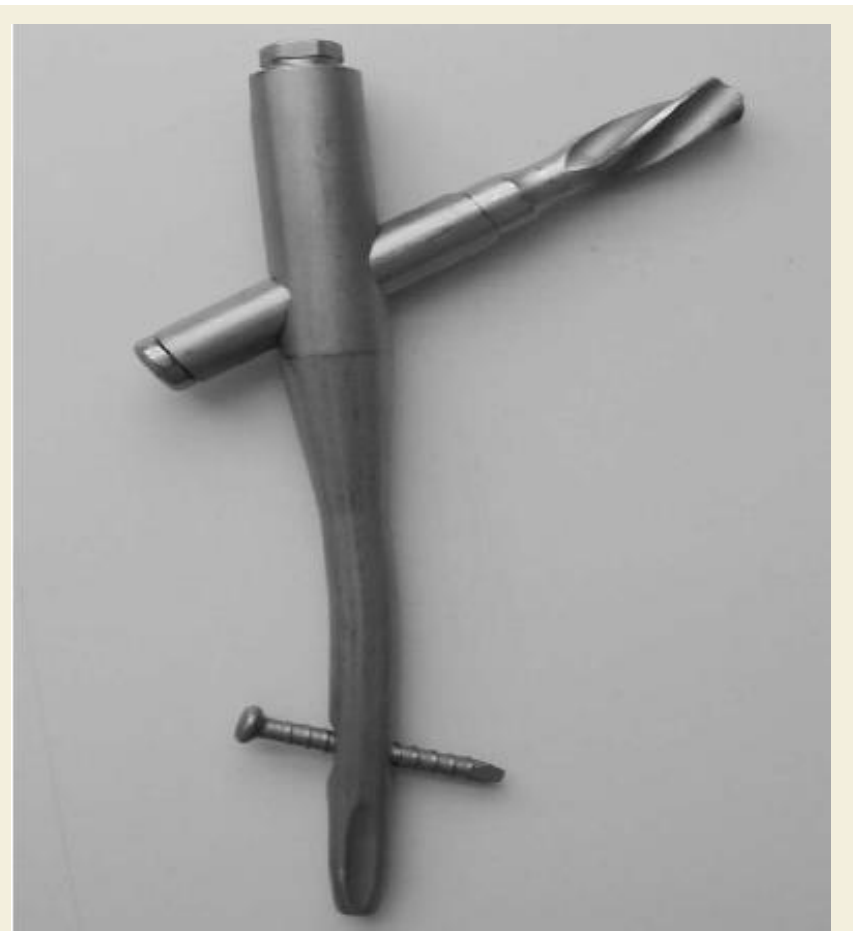

Figure 1: The standard proximal femoral nail with helical blade (PFNA) used in our patients, showing the helical blade and clothespin like distal ending, along with filled compression screw.

leads to high failure rate and great surgical trauma [10]. However, the best technique for managing such fractures is still controversial. This study revealed the post-operative outcome of using PFNA with helical blade more specifically rate of mechanical failure and any relation with specific patient characteristic (Figure 1). Fracture fragment assembly poses the greater goal of these surgeries; whereas this assembly's strength is determined by 5 variables: bone quality, fracture geometry, quality of reduction, implant design and implant placement [11].

Here, the intertrochanteric fracture patients who underwent open reduction and fixation with this PFNA technique were mostly elderly female with hypertension, which increases their propensity to fall (Table 1). In addition, the fractures were mostly due to low-impact injury with osteoporosis being previously diagnosed in around $20 \%$ of our patients. Around $60 \%$ of their fractures were unstable. The mean age of our patients, $80 \pm 10$ years, matched closely the rate noted in literature for such injuries with 80.8 years and 81.8 years old noted for inter/peritrochanteric fractures and unstable inter/peritrochanteric fractures respectively with a majority of female patients [12].

In the literature, many radiological parameters, such as TAD and neck shaft angle, are used to assess the quality of reduction trying to predict the rate of mechanical failure to help surgeons in controlling clinical outcomes. Our patients showed a bimodal distribution of TAD where most of the values were scattered between 23 and $24 \mathrm{~mm}$ and between 29 and $31 \mathrm{~mm}$, respectively (Figure 3). The neck shaft angle was noted to have a mean $\left(133 \pm 5.6^{\circ}\right)$ close to the upper border of the normal range (125-135 degrees) with a wide range from $116^{\circ}$ to $140^{\circ}$ (Figure 4). In addition, 35\% had displaced lesser trochanter and $36 \%$ had a displaced femoral shaft. These values indicate a substantial variability in the quality of reduction attained.

Despite this variability in thequality of reduction, there were no cases of intraoperative or postoperative mechanical failure in our patients. Thus, the postoperative radiological outcomes used in our study (TAD, neck shaft angle, displacement of lesser trochanter and displacement of femoral shaft) did not predict the rate of mechanical failure. Hence, the quality of reduction whether it is optimal (i.e. TAD of $25 \mathrm{~mm}$, neck shaft angle of $130^{\circ}$, non-displaced lesser trochanter and femoral shaft) or suboptimal did not correlate with the postoperative outcomes of the intertrochanteric reduction (Figure 5).

Conversely, several studies showed a statistical and clinical significant relation between these radiological parameters and the rate of mechanical failure for other implants. When using Gamma nail and DHS,TAD $<25 \mathrm{~mm}$, avoidance of anterior hip screw placement and fracture reduction with a valgus neck shaft angle of $5-10^{\circ}$ (p-value $\left.=0.091\right)$ relative to the contralateral side was associated with a trend towards a lower rate of screw cut-out in intertrochanteric fixation [10]. Also, Hsueh et al demonstrated that a bad reduction (i.e. varus or moderately valgus neck shaft angle on AP view, $>20$ degrees angulation on the lateral view and $>4 \mathrm{~mm}$ displacement on either view) is associated with higher rate of the cutout (p-value $<0.033$ ) using DHS [13]. Also, the higher the neck shaft

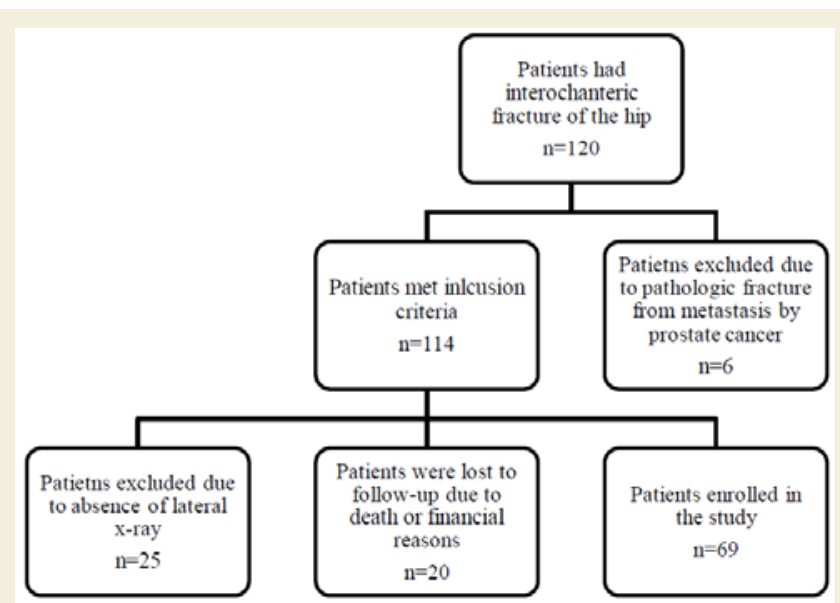

Figure 2: Flowchart illustrating selection of patients.

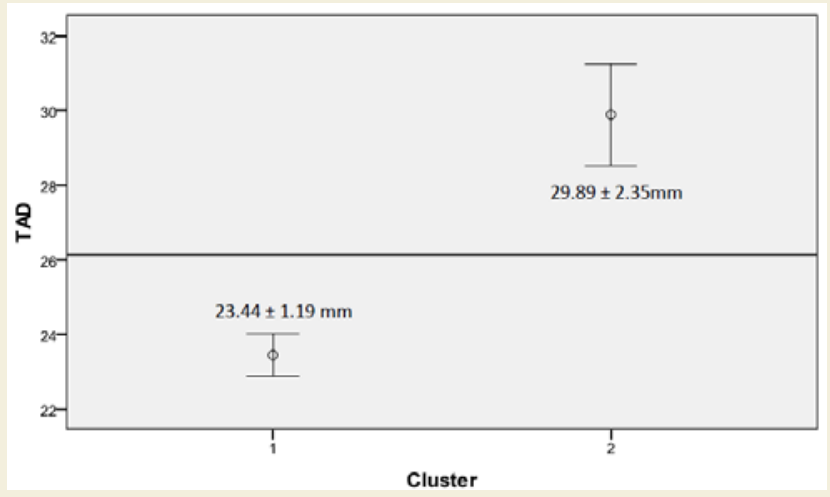

Figure 3: Tip Apex Index (TAD) can be classified into two clusters with a confidence interval of $95 \%$. The reference line presents the overall mean of $26.14 \pm 3.66 \mathrm{~mm}(n=43)$. 


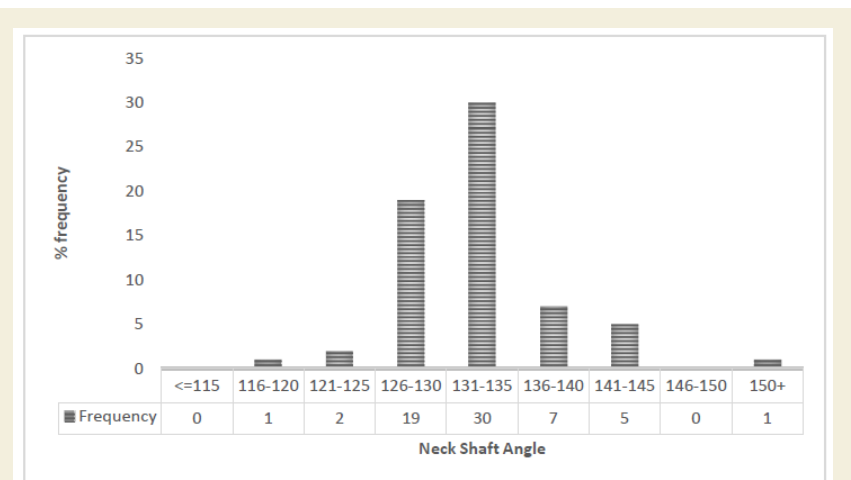

Figure 4: Frequency of neck shaft angle where most values are distributed over a range of 116 and $140(n=65)$.

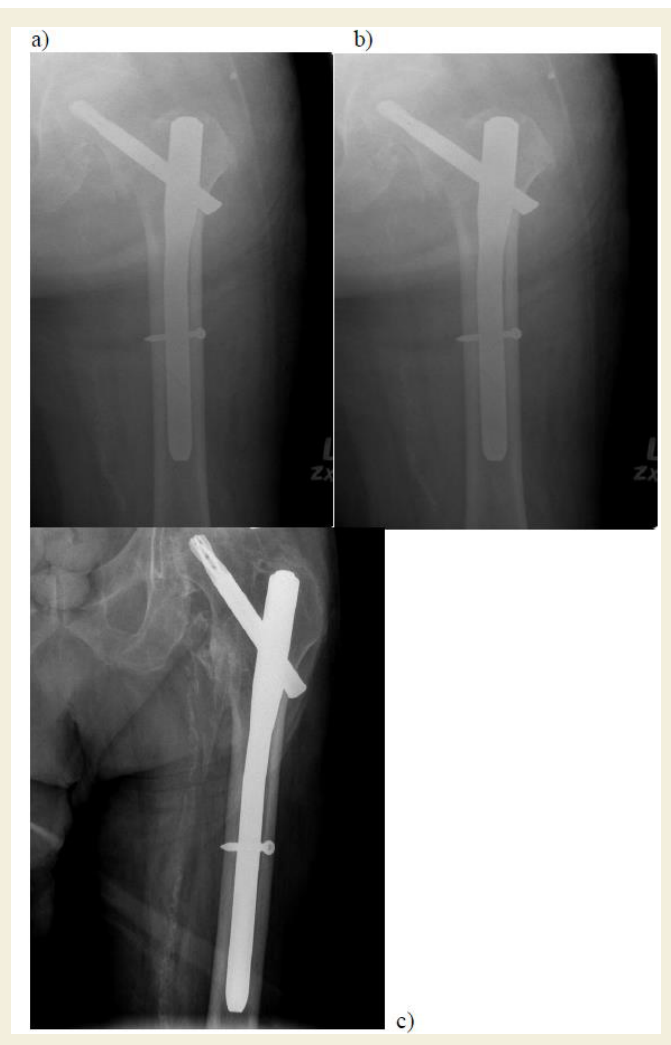

Figure 5: Post-operative radiographs of a patient who under went reduction and fixation with PFNA after intertronchanteric fracture: a) post-operative day 1 showing $T A D=44 \mathrm{~mm}$, neck shaft angle of $142^{\circ}$, and a displaced lesser trochanter and femoral shaft; b) 1 month and c) 3 months after surgery showing callus formation.

angle was associated with the easier sliding of the screw and fracture site impaction resulting in less cut-out $[14,15]$. In addition, multiple radiological parameters (improper entry points, posterior leg screw axis, mean angle between lag screw and femoral neck axis of $(-16.8)$, posterior displacement of the proximal fragment) are statistically and clinically significant predictors of cut-out using Gamma3 nail system [9]. Finally, one study reported that the TAD is the most accurate predictor of mechanical failure using Gamma nail [8].

Consequently, what might explain the absence of mechanical failure in our case despite the difference in radiological outcomes and type of fracture is the design of nail that is being used. Compared to PFNA, first generation Gamma nail combined intramedullary nailing and lag screw. Nevertheless, high complication rate was reported using the later implant due in the part of its design: which was discussed extensively by Radford et.al who compared postoperative complications in patients treated with Gamma nail and Dynamic Hip Screw. Preoperative and postoperative femoral fracture did occur with Gamma nail. One explanation to the preoperative fracture is the $12 \mathrm{~mm}$ diameter of the nail which leads to a higher stress on the medullary canal, increasing the risk of the fracture. But for the PFNA used in this study, the nail diameter was $10 \mathrm{~mm}$ which decreased the stress over the internal cortex hence, diminishing the risk of shaft fracture. Moreover due to its $10^{\circ}$ valgus curvature, first generation Gamma nail did not match the shape of the proximal part of the femoral shaft leading to misalignment of the femoral shaft axis with the Gamma nail axis. This causes a 3 point fixation in the femoral shaft, leading to stress concentration at these particular points, exacerbating the risk of fracture [10]. This excessive curvature leads to impingement of the tip of the nail against the external femoral cortex aggravating stress and increasing the risk of fracture [14]. On the other hand, PFNA used in this study has a $6^{\circ}$ valgus curvature, enabling a good fixation of the nail tip into the greater trochanter. This is when the second generation Gamma nail has been introduced with several corrections to decrease the rate of femoral shaft fracture: including a $4^{\circ}$ valgus curvature and smaller leg screw and nail diameter. However, Utrilla AL et al. reported other complications (lagcut-out, trochanteric fracture, DVT) comparable to the use of DHS that occurred with the second generation Gamma nail. In this study, there was no case of lagcut-out, trochanteric fracture or DVT [16]. In 2003, third generation was created with some modifications to decrease the likelihood of mechanical failure. Nevertheless, MingoRobinet J et al. compared the second generation with third generation gamma nail showing a higher cut-out rate with third generation gamma nail $(p$-value $<0.01)$ irrespective of radiological parameter TAD ( $p$-value $=0.4$ ) [17]. This difference in cut-out was attributed to the new lag screw design [15].

Yet, the best implant for intertrochanteric fracture is still unknown, but several studies compared the effectiveness of the PFNA with other implants used in the intertrochanteric fracture fixation

Table 1: Baseline characteristics for patients $(n=69)$ who underwent reduction and fixation with PFNA.

\begin{tabular}{|c|c|c|}
\hline \multicolumn{2}{|c|}{ Patients' Characteristics } & Frequencies (\%) \\
\hline \multicolumn{2}{|l|}{ Age (mean $\pm S D)$} & $80.3 \pm 10$ \\
\hline \multicolumn{2}{|c|}{ Weight (mean $\pm S D$ ) } & $72.1 \pm 15.6$ \\
\hline \multirow{2}{*}{ Gender } & Male & $27(39.1)$ \\
\hline & Female & $42(60.9)$ \\
\hline \multicolumn{2}{|l|}{ Smoking } & $22(36.7)$ \\
\hline \multicolumn{2}{|l|}{ Cancer } & $3(4.6)$ \\
\hline \multicolumn{2}{|l|}{ Osteoporosis } & $13(18.8)$ \\
\hline \multicolumn{2}{|l|}{ DM } & $28(43.1)$ \\
\hline \multicolumn{2}{|l|}{$\mathrm{DL}$} & $22(33.8)$ \\
\hline \multicolumn{2}{|l|}{ CAD } & $22(33.8)$ \\
\hline \multicolumn{2}{|l|}{ HTN } & $35(53.8)$ \\
\hline \multicolumn{2}{|l|}{ Renal } & $5(7.7)$ \\
\hline \multirow{3}{*}{ ASA } & 2 & $7(11.7)$ \\
\hline & 3 & $40(66.7)$ \\
\hline & 4 & $13(21.7)$ \\
\hline
\end{tabular}


Citation: Naja AS, Hanna T, Haidar R, Tamim H, Saghieh S. Predictors of Mechanical Failure Rate in Reduction of Intertrochanteric Fracture of the Femur Using Proximal Femoral Nail with Helical Blade. J Orthopedics Rheumatol. 2018; 5(1): 5

ISSN: 2334-2846

Table 2: Displacement of lesser trochanter/femoral shaft displacement and their relation with the different baseline characteristics of the patients.

\begin{tabular}{|c|c|c|c|c|c|c|c|}
\hline & & $\begin{array}{l}\text { Displaced lesser } \\
\text { tronchanter }(n=24)\end{array}$ & $\begin{array}{l}\text { Nondisplaced lesser } \\
\text { tronchanter }(n=40)\end{array}$ & $p$-value & $\begin{array}{l}\text { Displaced femoral } \\
\text { shaft }(n=19)\end{array}$ & $\begin{array}{c}\text { Nondisplaced femoral } \\
\text { shaft }(n=44)\end{array}$ & $p$-value \\
\hline \multicolumn{2}{|c|}{ Age $($ mean $\pm S D)$} & $78.4 \pm 13.1$ & $82.7 \pm 6.4$ & 0.24 & $81.9 \pm 6.6$ & $80.4 \pm 11.1$ & 0.41 \\
\hline \multirow{2}{*}{ Gender } & Male & $11(50.0 \%)$ & $13(34.2 \%)$ & & $7(38.9 \%)$ & $16(39.0 \%)$ & \\
\hline & Female & $11(50.0 \%)$ & $25(65.8 \%)$ & 0.23 & $11(61.1 \%)$ & $25(61.0 \%)$ & 0.99 \\
\hline \multicolumn{2}{|c|}{ Smoking } & $12(54.5 \%)$ & $26(70.3 \%)$ & 0.23 & $14(77.8 \%)$ & $24(60.0 \%)$ & 0.19 \\
\hline \multicolumn{2}{|l|}{ Cancer } & $1(4.2 \%)$ & $1(2.5 \%)$ & 0.71 & $1(5.3 \%)$ & $1(2.3 \%)$ & 0.53 \\
\hline \multicolumn{2}{|c|}{ Osteoporosis } & $4(33.3 \%)$ & $8(66.7 \%)$ & 0.63 & $4(33.3 \%)$ & $8(66.7 \%)$ & 0.78 \\
\hline \multicolumn{2}{|l|}{ DM } & $11(45.8 \%)$ & $16(40.0 \%)$ & 0.65 & $10(52.6 \%)$ & $17(38.6 \%)$ & 0.3 \\
\hline \multicolumn{2}{|l|}{$\mathrm{DL}$} & $9(37.5 \%)$ & $12(30.0 \%)$ & 0.54 & $6(31.6 \%)$ & $15(34.1 \%)$ & 0.85 \\
\hline \multicolumn{2}{|l|}{ HTN } & $15(62.5 \%)$ & $19(47.5 \%)$ & 0.24 & $10(52.6 \%)$ & $23(52.3 \%)$ & 0.98 \\
\hline \multicolumn{2}{|l|}{ Renal } & $2(8.3 \%)$ & $3(7.5 \%)$ & 0.9 & $1(5.3 \%)$ & $4(9.1 \%)$ & 0.61 \\
\hline \multirow[b]{2}{*}{ ASA } & 2 & $4(17.4 \%)$ & $3(8.3 \%)$ & & $6(15.0 \%)$ & $6(15.0 \%)$ & \\
\hline & 3 & $15(65.2 \%)$ & $24(66.7 \%)$ & & $24(60.0 \%)$ & $24(60.0 \%)$ & \\
\hline
\end{tabular}

Table 3: Summary of studies in which PFNA was compared to other types of screws and/or nails.

\begin{tabular}{|c|c|c|c|c|}
\hline Reference & $\begin{array}{l}\text { Number of patients or } \\
\text { studies }\end{array}$ & Study design & $\begin{array}{l}\text { Types of nails } \\
\text { included }\end{array}$ & Outcomes \\
\hline Yaozeng $X$ et al. 2010 [20] & 107 & Comparative study & PFNA vs. TGN & PFNA provides less blood loss and less fluoroscopy time \\
\hline Liu Y et al. 2010 [22] & 169 & Retrovspective cohort & PFNA & $\begin{array}{l}\text { PFNA is safe, user friendly and less invasive than other } \\
\text { types of nails }\end{array}$ \\
\hline Shen L et al. 2013 [21] & $5 \mathrm{RCT}$ & Meta-analysis & PFNA vs. DHS & $\begin{array}{l}\text { PFNA showed less blood loss and fewer complication time } \\
\text { compared with DHS }\end{array}$ \\
\hline Ana M et al. 2011 [24] & 208 & $\begin{array}{l}\text { Prospective randomized } \\
\text { trial }\end{array}$ & PFNA vs. PFN & PFNA showed lower incidence of cutout compared to PFN \\
\hline Ma KL et al. 2014 [3] & 14 studies & Meta-analysis & $\begin{array}{l}\text { PFNA vs. DHS vs. } \\
\text { Gamma nail }\end{array}$ & $\begin{array}{l}\text { PFNA showed minimal rate of fixation failure, less blood } \\
\text { loss, and shorter hospital stay compared to DHS and } \\
\text { Gamma nail }\end{array}$ \\
\hline Xu YZ et al. 2010 [23] & 106 & $\begin{array}{l}\text { Prospective randomized } \\
\text { study }\end{array}$ & PFNA vs. DHS & $\begin{array}{l}\text { PFNA allowed earlier mobilization and faster recovery than } \\
\text { the DHS }\end{array}$ \\
\hline
\end{tabular}

(Tables 1-3). PFNA provides less blood loss and shorter fluoroscopy time compared to gamma nail and DHS [3,18-21]. Also, it had shorter fluoroscopy time as compared to third generation gamma nail and fewer complications as compared to DHS and a faster recovery as compared to both [21-23]. In addition, minimal failure rate noted for PFNA as compared to others and it even showed less cut out rate (1.9\%) compared to PFN (4.8\%) attributing it to the superiority of blade compared to leg screw in the stabilization of bone stock [24].

The noted advantages can be attributed to the following mechanical properties of the proximal femoral nail with helical blade used in this study:

1. The helical blade that one can insert in via bone compaction and necessitate less bone removal, then a leg screw gives an angular and rotational stability especially in unstable fracture and in osteoporotic patients decreasing the risk of head and neck fracture [7].

2. The helical blade rather than screw allows better insertion in the femoral head potentially limiting the postoperative cut-out due to various deviation and rotation.

3. The larger surface area of the blade allows a more stable fixation in terms of axial loading especially in severe osteoporotic a wider distribution of forces within the place of highest bone density decreasing the risk of fracture [6].

4. The $6^{\circ}$ valgus curvature of this nail allows an excellent fit of the nail-tip to the greater trochanter limiting intraoperative femoral shaft fracture as seen in our results.

5. The $10 \mathrm{~mm}$ distal diameter allowed an optimal fit in the medullary canal without inducing excessive stress on the wall and without the need of hammer use or the excessive reaming that weakens the medullary canal and increases the risk of intraoperative femoral shaft fracture.

Thus, this study did not support the previous recommendations about the importance of controlling the quality of reduction in limiting the rate of cut out and intraoperative femoral fracture. But, the design of the intramedullary implant (i.e. PFNA) that fits the anatomy of the fractured femur might be the most influential determinant of mechanical failure rate, irrespective of reduction quality.

Trying to reduce the fracture in an excellent fashion will expose the patient to longer operative time and higher amount of anesthetics putting the patient at risk of many complications (infections, deep vein thrombosis, urinary retention, urinary tract infection, etc.). Thus, as per our study, using PFNA in reducing and fixing the intertrochanteric fracture even without ensuring optimal reduction, can provide fracture assembly and reduce the rate of cut out significantly during shorter operative time consequently indirectly resulting in lower cost and lower risk of complications.

There are three points merit to discuss. First, although some 
Citation: Naja AS, Hanna T, Haidar R, Tamim H, Saghieh S. Predictors of Mechanical Failure Rate in Reduction of Intertrochanteric Fracture of the Femur Using Proximal Femoral Nail with Helical Blade. J Orthopedics Rheumatol. 2018; 5(1): 5

studies reported that the rate of deep vein thrombosis ranged from $2 \%$ to $50 \%$ after hip fracture; yet, none of the patients in this study developed thrombosis. This could be due to the reason that all patients received thromboprophylaxis from admission that was continued for at least 10 days after the surgery. Second, osteoporosis could be a factor that can affect functional recovery and re-fracture rate after hip fracture surgery especially in elderly patients $[25,26]$. However, patients who had osteoporosis in this study did not have mechanical failure probably due to the surgical technique or small sample size. Third, early weight bearing could be a factor for dislocation thus hip surgery failure; nevertheless, this was not noted in this study probably due to the small number of patients [27].

However, our study has certain limitations. First, it is a retrospective study with the problems inherent with this methodology. Secondly, patient and surgeon related confounders may have existed. Third, it is possible that we did not address every potential confounding variable in our analyses. Fourth, due to a retrospective study, it is possible that variables contribute to complications were unaccounted for in this study. Fifth, the follow-up period was relatively short. However, most of cut-outs usually occur within 3 months. Sixth the sample population is taken from one medical center with a limited number of patients which might not be presenting the entire population. Sixth we did not compare our results with another implant to analyze the advantages of PFNA over the same sample of the population to limit as many confounders as possible. Seventh we did not analyze the different entry points of the implant which is one of the potential predictors of mechanical failure.

\section{Conclusion}

Most of the literature did conclude a superiority of proximal femoral nail with helical blade in reducing orthopedic and nonorthopedic complications that were attributed to both the philosophy of the implant and quality of reduction. However, superiority of proximal femoral nail with helical blade regardless of the reduction quality (i.e. position of the blade, neck shaft angle, and lesser trochanter and femoral shaft displacement) was noted. Thus, the findings suggested that the design of the proximal femoral nail with helical blade offers a higher stability and decrease in rotational mobilization, which might be the potential preventer of mechanical failure in this study. The use of PFNA with helical blade, irrespective of reduction quality, might decrease operative time and cost. Larger clinical trials are needed to further demonstrate this concept.

\section{References}

1. Sadic S, Custovic S, Jasarevic M, Fazlic M, Smajic N, et al. (2014) Proximal femoral nail antirotation in treatment of fractures of proximal femur. Med Arh 68: 173-177

2. Kulkarni GS, Limaye R, Kulkarni M, Kulkarni S (2006) Intertrochanteric fractures. Indian J Orthop 40: 16-23.

3. Ma KL, Wang X, Luan FJ, Xu HT, Fang Y, et al. (2014) Proximal femoral nails antirotation, gamma nails, and dynamic hip screws for fixation of intertrochanteric fractures of femur: a meta-analysis. Orthop Traumatol Surg Res 100: 859-866.

4. Arirachakaran $A$, Amphansap $T$, Thanindratarn $P$, Piyapittayanun $P$, Srisawat $P$, et al. (2017) Comparative outcome of PFNA, gamma nails, PCCP, medoff plate, LISS and dynamic hip screws for fixation in elderly trochanteric fractures: a systematic review and network meta-analysis of randomized controlled trials. Eur J Orthop Surg Traumatol 27: 937-952.
5. Kristek D, Lovrić I, Kristek J, Biljan M, Kristek G, et al. (2010) The proximal femoral nail antirotation (PFNA) in the treatment of proximal femoral fractures. Coll Antropol 34: 937-940.

6. Lenich A, Vester H, Nerlich M, Mayr E, Stöckle U, et al. (2010) Clinical comparison of the second and third generation of intramedullary devices for trochanteric fractures of the hip--blade vs. screw. Injury 41: 1292-1296.

7. Mereddy P, Kamath S, Ramakrishnan M, Malik H, Donnachie N (2009) The AO/ASIF proximal femoral nail antirotation (PFNA): a new design for the treatment of unstable proximal femoral fractures. Injury 40: 428-432.

8. Geller JA, Saifi C, Morrison TA, Macaulay W (2010) Tip-apex distance of intramedullary devices as a predictor of cut-out failure in the treatment of peritrochanteric elderly hip fractures. Int Orthop 34: 719-722.

9. Tsai SW, Lin CJ, Tzeng YH, Lin CC, Huang CK, et al. (2017) Risk factors for cut-out failure of Gamma3 nails in treating unstable intertrochanteric fractures: an analysis of 176 patients. J Chin Med Assoc 80: 587-594.

10. Andruszkow H, Frink M, Frömke C, Matityahu A, Zeckey C, et al. (2012) Tip apex distance, hip screw placement, and neck shaft angle as potential risk factors for cut-out failure of hip screws after surgical treatment of intertrochanteric fractures. Int Orthop 36: 2347-2354.

11. Kaufer H (1980) Mechanics of the treatment of hip injuries. Clin Orthop Relat Res: 53-61.

12. Lakstein D, Hendel D, Haimovich Y, Feldbrin Z (2013) Changes in the pattern of fractures of the hip in patients 60 years of age and older between 2001 and 2010: a radiological review. Bone Joint J 95-B: 1250-1254.

13. Hsueh KK, Fang CK, Chen CM, Su YP, Wu HF, et al. (2010) Risk factors in cutout of sliding hip screw in intertrochanteric fractures: an evaluation of 937 patients. Int Orthop 34: 1273-1276.

14. Loch DA, Kyle RF, Bechtold JE, Kane M, Anderson K, et al. (1998) Forces required to initiate sliding in second-generation intramedullary nails. $\mathrm{J}$ Bone Joint Surg Am 80: 1626-1631.

15. Meislin RJ, Zuckerman JD, Kummer FJ, Frankel VH (1990) A biomechanical analysis of the sliding hip screw: the question of plate angle. J Orthop Trauma 4: $130-136$

16. Utrilla AL, Reig JS, Muñoz FM, Tufanisco CB (2005) Trochanteric gamma nail and compression hip screw for trochanteric fractures: a randomized, prospective, comparative study in 210 elderly patients with a new design of the gamma nail. J Orthop Trauma 19: 229-233.

17. Mingo-Robinet J, Torres-Torres M, Martínez-Cervell C, Alonso Del Olmo JA, Rivas Laso JA, et al, (2015) Comparative study of the second and third generation of gamma nail for trochanteric fractures: review of 218 cases. J Orthop Trauma 29: e85-e90.

18. D’Arrigo C, Carcangiu A, Perugia D, Scapellato S, Alonzo R, et al. (2012) Intertrochanteric fractures: comparison between two different locking nails. Int Orthop 36: 2545-2551.

19. Stern R, Lübbeke A, Suva D, Miozzari H, Hoffmeyer P (2011) Prospective randomised study comparing screw versus helical blade in the treatment of low-energy trochanteric fractures. Int Orthop 35: 1855-1861.

20. Yaozeng X, Dechun G, Huilin Y, Guangming Z, Xianbin W (2010) Comparative study of trochanteric fracture treated with the proximal femoral nail antirotation and the third generation of gamma nail. Injury 41: 1234-1238.

21. Shen L, Zhang Y, Shen Y, Cui Z (2013) Antirotation proximal femoral nail versus dynamic hip screw for intertrochanteric fractures: a meta-analysis of randomized controlled studies. Orthop Traumatol Surg Res 99: 377-383.

22. Liu Y, Tao R, Liu F, Wang Y, Zhou Z, et al. (2010) Mid-term outcomes after intramedullary fixation of peritrochanteric femoral fractures using the new proximal femoral nail antirotation (PFNA). Injury 41: 810-817.

23. Xu YZ, Geng DC, Mao HQ, Zhu XS, Yang HL (2010) A comparison of the proximal femoral nail antirotation device and dynamic hip screw in the treatment of unstable pertrochanteric fracture. J Int Med Res 38: 1266-1275.

24. Ana M, Isidro M, Miquel R, Werner B, Antonio C, et al. (2018) PFN versus PFNA in treatment of trochanteric femoral fractures: a prospective study. 
Citation: Naja AS, Hanna T, Haidar R, Tamim H, Saghieh S. Predictors of Mechanical Failure Rate in Reduction of Intertrochanteric Fracture of the Femur Using Proximal Femoral Nail with Helical Blade. J Orthopedics Rheumatol. 2018; 5(1): 5.

\section{ISSN: $2334-2846$}

Orthop Proc 93-B.

25. McNamara I, Sharma A, Prevost T, Parker M (2009) Symptomatic venous thromboembolism following a hip fracture: incidence and risk factors in 5,300 patients. Acta Orthop 80: 687-692.

26. Makridis KG, Karachalios T, Kontogeorgakos VA, Badras LS, Malizos KN (2015) The effect of osteoporotic treatment on the functional outcome, re- fracture rate, quality of life and mortality in patients with hip fractures: a prospective functional and clinical outcome study on 520 patients. Injury 46 : 378-383.

27. Jung J, Schmid NV, Kelm J, Schmitt E, Anagnostakos K (2009) Complications after spacer implantation in the treatment of hip joint infections. Int J Med Sci 6: 265-273. 